\title{
Exogenous calcium protects postweanling rats from salicylate-induced changes in auditory conditioning
}

\author{
JAMES F. BRENNAN and ALEX WHIFFEN-MANSFIELD \\ University of Massachusetts, Boston, Massachusetts \\ and \\ PAWEL J. JASTREBOFF \\ Tinnitus and Hyperacusis Center, University of Maryland School of Medicine \\ Baltimore, Maryland
}

\begin{abstract}
During Days 26-32 (postnatal), 18 rats drank more of a 0.05-M calcium-enriched solution when it was given to them ad lib or $1 \mathrm{~h}$ daily, but not when it was given 15 min daily, compared with 18 pups with similar access to plain water only. In a second experiment, acquisition and extinction of auditory conditioned suppression were observed in thirty-six 25-day-old pups, half of them reared with the calcium supplement throughout gestation and preweanling, and the other half reared with tap water. Twenty-two hours before training, the subjects were fluid deprived and exposed to continuous noise of $60 \mathrm{~dB}(\mathrm{SPL})$. Two hours prior to each session, the subjects received either injections of sodium salicylate before both sessions ( $n=6$ for each fluid type), saline before acquisition and salicylate before the extinction session $(n=6)$, or saline before both sessions $(n=6)$. Suppression of the approach to drink at the end of an alleyway paired the offset of the 60-dB noise and footshock. Calcium afforded subjects protection from salicylate-induced changes in auditory behavior during both acquisition and extinction.
\end{abstract}

In several reports, we have proposed an animal model of phantom sound perception produced by salicylate and quinine to determine both behavioral (Brennan \& Jastreboff, 1989; Brennan \& Jastreboff, 1991; Jastreboff, Brennan, Coleman, \& Sasaki, 1988) and electrophysiological effects (Jastreboff, Brennan, \& Sasaki, 1987; Chen \& Jastreboff, 1995). Following the determination of salicylate acid uptake in blood serum, cerebrospinal fluid (CSF), and perilymph (PL) under various experimental conditions (Jastreboff, Issing, Brennan, \& Sasaki, 1988), we have found that salicylate reliably induces phantom auditory sensations in adult (Jastreboff, Brennan, et al., 1988) and young (Brennan, Byrnes, \& Jastreboff, 1993) pigmented rats.

Our basic experimental demonstration of the behavioral measure of subjective auditory sensations (Jastreboff, Brennan, et al., 1988) used a conditioned lick suppression paradigm to measure salicylate-induced effects that depended on the time of initial onset of the subjective auditory effect. We have since replicated this finding and found it resistant to changes in motivation and variation in auditory context (Jastreboff \& Brennan, 1992). Additionally, the effect was absent when visual stimuli

This work has been supported by Grant NIH NIDCD R01 DC00299. Requests for reprints should be addressed to J. F. Brennan, Graduate School, Loyola University Chicago, 6525 North Sheridan Road, Chicago, IL 60626. were used, indicating that the salicylate-induced effect is specifically auditory (Jastreboff, Brennan, et al., 1988; Jastreboff, Brennan, \& Sasaki, 1991). Recently, we reported a linear dose-response relationship, which we used to approximate the subjective loudness of the salicylateinduced sound by equivalent behaviors to discrete tones of varying intensities (Jastreboff \& Brennan, 1994b).

The collective impact of these data supports a theoretical model of phantom sound that emphasizes the role of calcium and calcium-channel involvement (Jastreboff, 1990; Jastreboff, Nguyen, Brennan, \& Sasaki, 1992). In the normally functioning cochlea, calcium can interfere with the transduction of sound through several mechanisms: (1) the position of the tectorial membrane (Kronester-Frei, 1979); (2) the calcium-dependent potassium channels of hair cells (Hudspeth, 1986); (3) the mechanical properties of hair-cell cilia (Brownell, Shehata, \& Imredy, 1990); (4) the slow motile properties of outer hair cells (Flock, Flock, \& Ulfendahl, 1986); and (5) the release of neurotransmitter(s) from hair cells (Jastreboff, 1990). We have found that salicylate decreases free calcium in the PL, and that supplementing the adult diet with exogenous calcium attenuates salicylate effects on free perilymphatic calcium at the behavioral level (Jastreboff et al., 1992).

In terms of the behavioral evaluation of calcium's attenuation of salicylate-induced sound perception, we trained 36 rats to suppress drinking upon the offset of a continuous background noise-the conditioned stimulus (CS)-paired with a pulse of footshock- the uncondi- 
tioned stimulus (US; Jastreboff et al., 1992). For half of the subjects, the ad-lib drinking water and the fluid available in the experimental chamber were replaced by a $0.05-\mathrm{M}$ solution of $\mathrm{CaCl}_{2}$. Four groups of subjects $(n=6$ per group) were injected s.c. with $350 \mathrm{mg} / \mathrm{kg}$ sodium salicylate either before (one group with access to water; one group with access to water + calcium) or after (one group with access to water; one group with access to water + calcium) suppression training. As mentioned above, this type of temporal relationship between salicylate introduction and behavioral training has resulted in opposing salicylate effects on extinction of suppression (Jastreboff, Brennan, et al., 1988). In contrast to the salicylate groups that drank plain water and showed significantly faster extinction when injections began after suppression training and marked resistance to extinction when the injections began before training, the results of the groups drinking the calcium solution showed little salicylate effect. In parallel experiments, calcium-selective microelectrodes were inserted into the PL through the round window membrane, and measurements were made every $5 \mathrm{~min}$ before and after salicylate injection for up to $6 \mathrm{~h}$ (Jastreboff et al., 1992). The group given tap water exhibited a decrease of free calcium. Comparison with rats given the same exogenous calcium solution as in the behavioral experiment showed no change in the basefree calcium level, but a significant attenuation in the salicylate-induced free-calcium decrease. Finally, nimodipine, a blocker of L-type calcium channels, decreased, and in higher dose eliminated, the detection of salicylate-induced phantom sound perception (Jastreboff et al., 1992). Thus, these findings support the hypothesis that calcium homeostasis may be critical in normal hearing, and that its disturbance may lead to auditory disorders, including tinnitus. Recently, we have reported that exogenous calcium protects adult rats from the toxic effects of high salicylate doses when subjects are exposed to stress that is specifically auditory (Jastreboff \& Brennan, 1994a).

The major auditory event of the early preweanling period centers on ultrasounds emitted by the pup and recognized by the dam as a signal to locate the pup, in addition to supporting other functions, including temperature regulation (cf. Blumberg, Efimova, \& Alberts, 1992; Goodwin, Molina, \& Spear, 1994; Hofer, Brunelli, \& Shair, 1993). Beginning on Day 4 and reaching peaks of both rate and intensity between Days 10 and 18, ultrasounds then decrease as other sensory systems become functional (Sales \& Smith, 1978). The study of neural development underlying auditory functions suggests that the auditory system, like other sensory systems, undergoes dramatic change during this period. The auditory meatus begins to open by Day 12 and is fully open at around Day 14 (Kelly, 1990; Rubel, 1984; Rubel, Hyson, \& Durham, 1990). Recordings from the developing auditory nerve show changes in threshold levels and tuning curves of auditory nerve fibers, increases in the dynamic ranges of rate and intensity, and rapid decreases in the latencies of response (Brugge, 1986). Moreover, behav- ioral evidence of proactive reflexive and associative influences from auditory stimuli exposed to rat pups as young as 14 days of age and tested at Day 18 (Hyson \& Rudy, 1984; Rudy \& Hyson, 1982, 1984) supports Rubel's $(1978,1984)$ notion that the place principle of cochlear sensitivity (Békésy, 1960) may shift during early cochlear functioning up to 18-20 days of age. More recently, Friauf (1992) used the expression of $c$-fos protooncogene to mark the frequency-place map in developing and adult rats. From Day 14, both low and high auditory frequencies induced strong Fos immunoreactivity in seven brain-stem nuclei, and the tonotopic organization assumed an adultlike configuration. One possible explanation of the lack of evidence from the method of $c$-fos immunocytochemistry of the shift in frequencyplace reported from behavioral experiments may be that while $c$-fos expression is induced, the neurons are still not sufficiently mature to generate action potentials.

One of the major issues raised by our experiments on salicylate-induced sound perception concerned whether the effect could be found at young age levels. To address this issue, two experiments used preweanling (14- to 21day-old) and postweanling (32-day-old) pups (Brennan et al., 1993). Adapting a behavioral approach-avoidance task sensitive to immature rats, we injected 9 groups of 5 rats (14-21 days old) in Experiment 1 with sodium salicylate or the saline vehicle either before or after acquisition of suppression of an approach response to a lactating dam. The sequence of injection initiation affected acquisition and extinction rates of subjects older than 17 days in the direction predicted from adult patterns, while these effects were not seen at the youngest age level. In addition, extended exposure to salicylate administration beginning at Day 14 did not reveal any chronic, deleterious effects on auditory mediated behavior in 20 preweanling pups when suppression training and testing occurred at Days 20 and 21. Using a similar task for 18 postweanling pups of 32 days of age in Experiment 2 resulted in the predicted effects of greater suppression and resistance to extinction when salicylate was administered prior to both acquisition and extinction sessions, while subjects receiving saline injections before training and salicylate after training extinction showed the most rapid recovery from suppression.

Since our studies of the effect of exogenous calcium supplement on salicylate-induced phantom sound perception with adult rats pointed to an attenuating effect of calcium supplement on salicylate-induced effects (Jastreboff et al., 1992), we wanted to determine the effects of calcium introduced earlier in development, as the auditory system becomes behaviorally functional.

\section{EXPERIMENT 1A Calcium Effects on Weight and Water Consumption}

To extend the study of an enriched calcium diet on attenuation of salicylate effects, we first needed to determine whether fluid intake and growth rates of mildly de- 
prived young subjects raised on a diet of water supplemented with calcium $\left(0.05 \mathrm{M} \mathrm{CaCl}_{2}\right)$ would differ from those of counterparts raised on normal tap water.

\section{Method}

A total of 36 male pigmented postweanling rat pups served in this experiment. The subjects were born in the animal colony of the University of Massachusetts at Boston and were progeny of Charles River strains. They were maintained on a 12:12-h light:dark cycle (lights on at 7:00 a.m.) throughout the experiment. From before mating, the prospective dams were allowed ad-lib access to either the plain tap water or the calcium solution, which continued throughout gestation, birth and preweaning. For the duration of the gestation and their preweanling periods, each dam and, eventually, each litter were housed in individual acrylic maternity cages $15 \mathrm{~cm}$ high $\times 22 \mathrm{~cm}$ wide $\times 44 \mathrm{~cm}$ long. During this time, the floor bedding was changed once on Day 2 postnatally, when litters were also sexed and culled to no more than 8 males. The subjects were weaned at 22 days of age, housed singly, and given access to the appropriate fluid: water for 18 pups, and the calcium solution for the other 18 subjects.

Weight change and liquid intake were monitored in all subjects from Day 23 through Day 32 of age. Beginning on Day 26, subgroups $(n=6)$ were assigned to one of three daily periods of access to the respective fluid solutions: $15 \mathrm{~min}$ or $1 \mathrm{~h}$ of restricted drinking or continued ad-lib drinking. The subjects were weighed at the same time each day-8:00 a.m.- when the two restricted periods of access to fluid also began. At the conclusion of the experiment, the subjects were euthanized with an overdose of nembutal and blood samples were drawn from their tails for analysis of calcium levels.

\section{Results and Discussion}

Calcium levels. The mean of the blood-serum calcium levels of 10 calcium-supplemented pups $(M=2.55 \mathrm{mM}$, $S E=0.099$ ) was greater than that of 12 water-reared pups $(M=2.12 \mathrm{mM}, S E=0.121)$. The difference between the groups sampled was significant $[t(20)=2.84, p<.01]$.

Consumption. Figure 1 shows the daily amount of fluid consumed across days for each combination of access and fluid. It should be noted that the limitation in access to the respective drinking fluids in four of the groups began on Day 26. A $2 \times 3 \times 10$ analysis of variance (ANOVA; Winer, 1971) of volume consumed by subjects revealed significant main effects for the type of fluid $[F(1,24)=$ $18.71, p<.0002]$, time of access $[F(2,24)=170.16$, $p<.0001]$, and progressive sessions $[F(9,216)=47.18$, $p<.0001]$. The latter main effects confirmed the obviously accelerated consumption shown in Figure 1 as the access time per day and the age of subjects increased across fluid type. All of the interactions among the independent variables were also significant. The fluid type $X$ access time interaction $[F(2,24)=3.47, p<.05]$, is reflected in the larger differences between the respective fluid groups in Figure 1 as access to drinking increased. That consumption of the calcium solution increased more than that of plain water across the experimental days was supported by the fluid type $\times$ progressive sessions interaction $[F(9,216)=6.69, p<.0001]$. The significant interaction of access time $\times$ progressive sessions $[F(18,216)=52.33, p<.0001]$ is most readily explained as the result of the abrupt drop in consumption
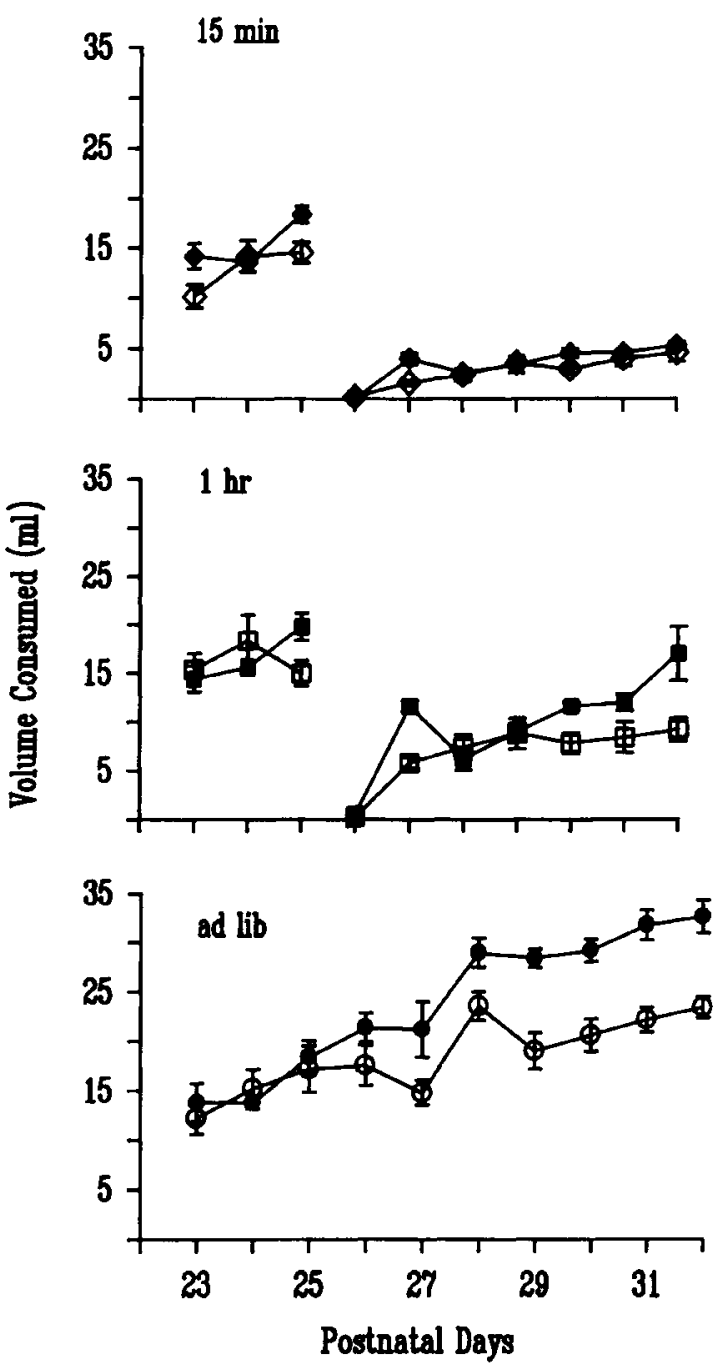

Figure 1. Mean consumption (ml) from Day 23 to Day 32 by subjects reared with the calcium-enriched solution (solid symbols) or the plain water (open symbols). Beginning on Day 26, four of the groups $(n=6)$ were limited to either 15 or $60 \mathrm{~min}$ of daily access to the respective fluids.

on the first day of limited access for four of the groups, and is also supported by the triple interaction of fluid type $\times$ access time $\times$ progressive sessions $[F(18,216)$ $=3.33, p<.001]$. The triple interaction indicated that, compared with plain-water intake, subjects consumed the calcium solution at a higher, increasing rate when permitted more than minimal access to fluid, and this trend for greater calcium consumption emerged only after several sessions. Duncan tests (all $p$ s $<.05$ ) confirmed that subjects with ad-lib access consumed more calcium-supplemented water than did their plain-water counterparts during the last six sessions, and that of the subjects with only $1-\mathrm{h}$ access, those consuming the calcium solution drank more than did those consuming plain water on Days 27 and 30-32. In the ad-lib access treatment, the highly linear trends were confirmed by 
significant regression coefficients within each respective fluid type of water and calcium $[r(8)=.852 ; r(8)=$ .969 ; both $p$ s $<.01]$.

Weight change. A similar analysis of the absolute weight changes from the levels at the first session showed that access time was again significant $[F(2,24)=141.32$, $p<.0001]$, with the expected finding that weight gain increased with increased opportunity to drink. While the main effect of fluid type was not significant, the interaction of fluid type $\times$ access time $[F(2,24)=7.54, p<$ .003 ] reflected differences in the relations between water and the calcium solution among the three access times. The effect of progressive sessions was significant $[F(9,216)=272.16, p<.0001]$, most probably reflecting growth in all of the pups. The interaction of access time $\times$ progressive sessions was also significant $[F(18,216)=$ $130.33, p<.0001]$, since weight gain increased fastest in the ad-lib groups and least in the 15-min-access condition. This interaction is also a product of the decline in weight on Day 27, following the initial deprivation day for the four limited-access groups. The triple interaction of fluid type $\times$ access time $\times$ progressive sessions $[F(18,216)=5.75, p<.0001]$, depicted in Figure 2, confirmed the greater weight gain under the ad-lib condition for drinking water than under the same condition for calcium on the last six sessions, while the trend was significant but reversed for the $1-\mathrm{h}$-access condition on Days 28, 30, and 32 (all $p$ s <.05).

Fluid consumed, supported only partially by weight gain, indicated that subjects tended to drink more of the calcium solution as deprivation and age increased, compared with counterparts drinking water only. The effect was clearest in the ad-lib groups, but was also present in the groups allowed access to fluids for $1 \mathrm{~h}$ per day; however, the trends in weight gain in the 1-h-access groups (Figure 2) were inconsistent with the consumption measure. Nevertheless, these data suggest that a calciumenriched diet does not seem to have detrimental effects on consumption, justifying the diet and the mild deprivation schedule used in the following experiment.

\section{EXPERIMENT 1B}

\section{Exogenous Calcium Effects on Interference From Salicylate-Induced Sound Perception in Conditioned Suppression}

In Experiment 1B, we proceeded to investigate whether exogenous calcium would protect young subjects from salicylate-induced auditory effects on acquired avoidance behavior. This experiment followed the procedure of the Brennan et al. (1993) study, and assessed acquisition of conditioned suppression of an approach response signaled by an auditory cue, followed by extinction of the suppression.

\section{Method}

Subjects. Thirty-six male, pigmented rat pups were born of dams given access to plain water or to the solution of $0.05 \mathrm{M}$ calcium chloride from the time of mating. Preweanling maintenance
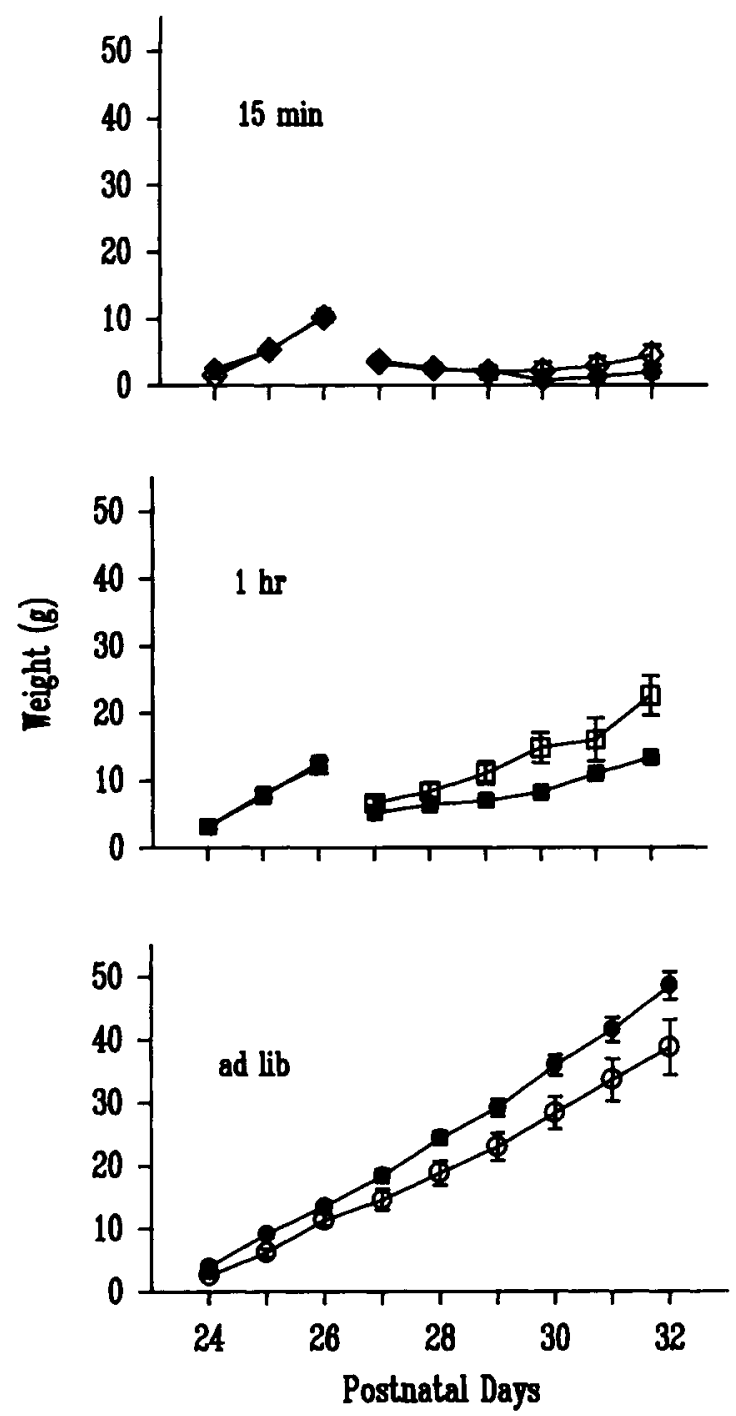

Figure 2. Mean weight gain from Day 23 for groups reared with either the calcium-enriched solution (solid symbols) or plain water (open symbols) and allowed limited (15- or 60-min) or ad-lib access to the respective solutions.

conditions were as described for Experiment 1A, and pups were weaned and housed singly at Day 22, and given ad-lib access to food and fluid. Half of the subjects continued to drink plain tap water, while the remaining 18 pups were given free access to the calcium solution. The subjects were 25 days of age at the time of the first behavioral training session, and their weights ranged from 59 to $71 \mathrm{~g}(M=63.14, S E=1.9)$; there were no systematic weight differences due to water- or water + calcium-rearing regimens. This finding is consistent with the pattern of weight change in the adlib access subjects of Experiment $1 \mathrm{~A}$, since group differences were negligible on Day 25 , and those differences that did emerge became pronounced with increasing age, contributing to the statistically significant effect in Experiment $1 \mathrm{~A}$.

Apparatus. Acquisition training and extinction testing were conducted in a straight-alley maze, $92 \mathrm{~cm}$ long $\times 16 \mathrm{~cm}$ wide $X$ $15 \mathrm{~cm}$ high. The walls and ceiling were made of clear acrylic, and the entire floor was composed of $2-\mathrm{mm}$ steel rods spaced $1 \mathrm{~cm}$ apart. A 14-cm-long start compartment was separated from the rest 
of the alleyway by a clear acrylic guillotine door, and the rods in the last $18 \mathrm{~cm}$ of the alley were wired to deliver a $1.0-\mathrm{mA}$ scrambled footshock from a BRS/LVE generator (Model SBS-004). The entire maze was suspended over a tray covered with hardwood bedding (Ab-sorb-dri). On the end wall, beyond the wired portion of the grid floor, a small hole $4 \mathrm{~cm}$ above the floor permitted insertion of a stainless steel drinking tube of the same variety as that used in the home cage. The tube was connected to a bottle that was suspended outside the maze and that dispensed the appropriate fluid. The ceiling of the maze was composed of a total of five hinged lids, providing access to a subject at any point in the maze. On two of the lids, 9-cm-wide Motorola speakers (P35-VAH) were mounted over perforated holes drilled through the plastic. Each speaker was centered $25 \mathrm{~cm}$ from each end of the maze. The experiment was conducted in an isolated room that attenuated external sounds, and the only illumination within the room was provided by a $25-W$ houselight aimed away from the apparatus but sufficiently bright to permit videotaping of each trial by a Sharp Camcorder (Model VL-170U). Background-noise presentations in the experimental chambers and in the home cages prior to training were provided by Hewlett-Packard precision noise generators (Model 8057A). The sound-pressure levels in the center of the alleyway and at the center of $3 \times 3 \mathrm{~m}$ rack housing individual cages were measured by a precision sound level meter (Quest Electronics, Model 155) in $1 / 3$ octave bands (Quest Electronics $1 / 3$ octave filter, Model OB-133). The SPL level was calculated for frequencies of $1-16 \mathrm{kHz}$ according to standard procedures (Durrant \& Lovrinic, 1984), and it was maintained at $60 \mathrm{~dB}$ (SPL). A lightemitting diode measuring $1 \mathrm{~mm}$ in diameter was placed over the alleyway as an indicator of CS/US onset. This light was not visible to the subjects, but provided a cue for the onset of stimuli when the videotape of the sessions was reviewed.

Procedure. Twenty-two hours prior to the first training session, the 25-day-old pups were deprived of ad-lib access to either water or the calcium solution and were exposed to $60-\mathrm{dB}$ (SPL) continuous background noise. Two hours before the session, three groups of 6 subjects drinking plain water and three groups of 6 subjects exposed to the calcium solution received either s.c. injections of $300 \mathrm{mg} / \mathrm{kg}$ salicylate acid, recalculated from the dose of sodium salicylate (Groups SB), or the saline vehicle only (Groups SA and Groups $C$ ) before the training session. The pups were trained individually, and initial training involved a subject's running down the alley to access a drinking tube with either water or the calcium solution in the presence of the continuous $60-\mathrm{dB}$ noise generated through speakers fixed to the ceiling of the alley maze. The subjects were allowed about $5 \mathrm{sec}$ of access to the drinking tube, and acquisition of the instrumental response was defined as two consecutive trials on which the subjects proceeded steadily, without any pause in progress, to the drinking tube. Subsequent trials involved pairing the offset of the background noise (CS) $0.5 \mathrm{sec}$ prior to the subject's stepping onto the electrified $18-\mathrm{cm}$ area of the grid floor (US) just before the drinking tube. If the subject retreated from the grid area back toward the start compartment, the noise was reactivated; if it proceeded on the grid with the shock on, the noise remained off. If a subject remained on the grid for $10 \mathrm{sec}$ because of disorientation, the trial was automatically terminated. Acquisition of the suppression CR was defined as five consecutive pause durations of at least $5 \mathrm{sec}$ when a subject failed to step onto the electrified grid area. Subjects that did not meet the acquisition criterion within 25 trials were discarded from the experiment and replaced with naive pups; 2 subjects, both from the plain-water condition, were discarded for this reason. Following the acquisition training session, the subjects were allowed $30 \mathrm{~min}$ of access to the respective fluids in a holding cage, and then returned to the home cage, all in the presence of the background noise. On the second day of the experiment, extinction of the suppression and recovery of the approach response were tested. Two hours prior to the session, each subject was removed from the litter cage and weighed, and the four SB and SA groups were injected with salicylate, while the controls (Groups $C$ ) received a second saline injection. The extinction trials involved the same sequence of events as did the acquisition trials, but the shock was disconnected. Each session continued until five consecutive trials were recorded without any apparent pause of more than $1 \mathrm{sec}$ at the end of the alley before the $18-\mathrm{cm}$ area of wired grids. All trials were videotaped, and two experimenters reviewed tapes separately to record data on the pause responses. Measures of trials to acquisition and extinction criteria, trial latencies, and pause durations were recorded.

\section{Results}

Acquisition. All subjects easily met the criterion for acquisition of the instrumental running response within five trials and proceeded to suppression training beginning on the next trial. Figure 3 shows the mean number of trials to meet the suppression criterion of five consecutive trials on which the subject failed to step onto the electrified grid area for at least $5 \mathrm{sec}$. A factorial ANOVA (Winer, 1971) of the mean number of trials to reach the suppression criterion, with the saline-treated groups (SA and C) combined, revealed that the type of fluid reinforcement was significant $[F(1,32)=7.00, p<.008]$. This effect confirmed the trend in Figure 3 showing that the calcium groups acquired the suppression behavior in fewer trials than the waterreared subjects, although there was a greater difference in the variability between calcium groups, also shown in Figure 3. Neither the effect of salicylate treatment nor the interaction attained significance.

For the evaluation of trial latencies during acquisition of the suppression, the total numbers of trials for each subject were divided into six blocks, and mean latencies per sixths of trials were calculated. This procedure allowed the measurement of alley latencies (as well as pause

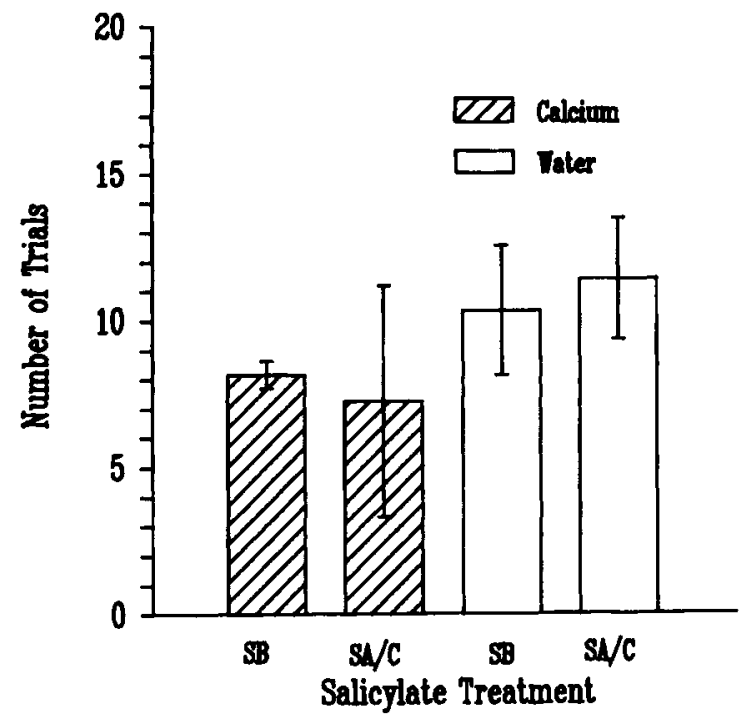

Figure 3. Mean number of trials to reach the suppression criterion of five consecutive pause responses in the groups reared and reinforced with exogenous calcium (slashed blocks) or plain water (open blocks). Prior to training under each reinforcement fluid, two groups received s.c. salicylate injection (SB), and the remaining four groups (SA/C) were injected with the saline vehicle. 
durations; see below) to be analyzed across all subjects at comparable points in suppression training, despite differing rates of acquisition. Figure 4 , in which blocks of training trials are represented by the mean trial latencies of all the trials in that block, shows the differences between calcium- (upper panel) and plain-water- (lower panel) reared subjects of each salicylate treatment (SB and combined SA/C groups). The measure of alley latency was defined as the time from the start of the trial until the onset of the auditory cue, and was analyzed in a mixed-design ANOVA (Winer, 1971) for the type of fluid reinforcement, salicylate drug group, and progressive blocks of trials. The significant main effect of fluid type $[F(1,32)=7.74, p<.009]$, supported the impression from Figure 4 that the plain-water-reared-and-reinforced subjects under both salicylate treatments (SB and combined SA/C) had somewhat overall shorter latencies. As with the measure of trials to acquisition of the suppression, there was no main effect observed for the salicylate drug treatment. Blocks of trials were significant $[F(5,160)$ $=2.87, p<.03$ ], indicating a fairly weak trend toward increased latencies as subjects were exposed to the US. Duncan tests revealed that a significant difference $(p<$ .05 ) between fluid rearing conditions emerged on the

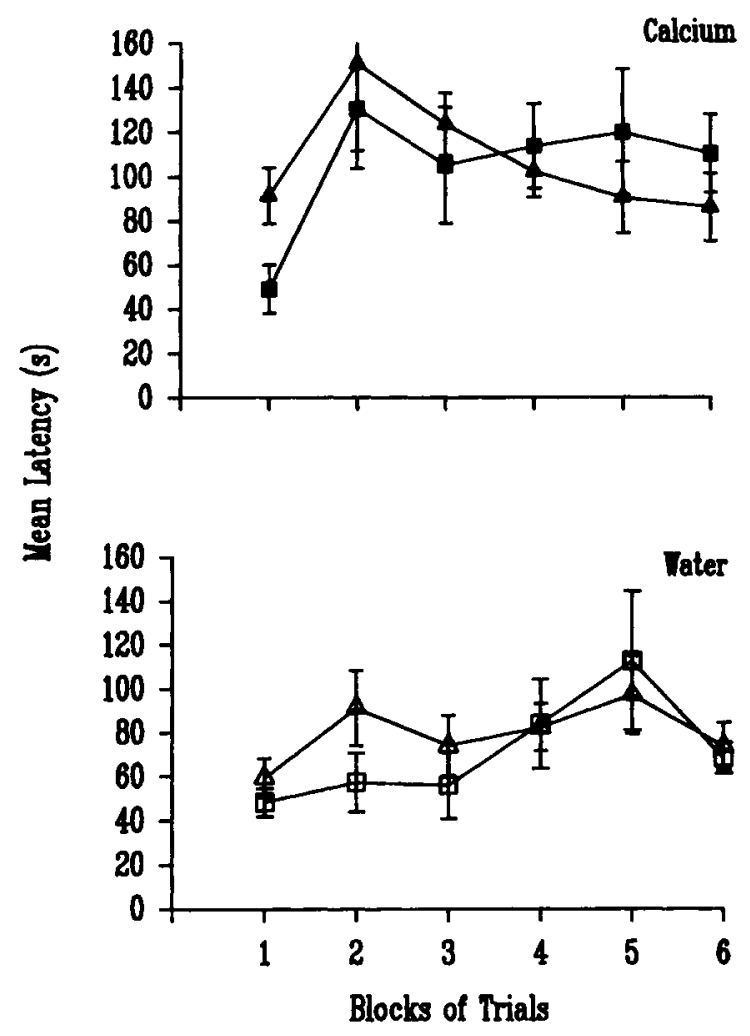

Figure 4. Mean alley latencies for each of six blocks of trials for subjects reared and reinforced with calcium-supplemented water (solid symbols) or plain water (open symbols). Within each fluid treatment, subjects received either injections of $300 \mathrm{mg} / \mathrm{kg}$ salicylate (SB groups, squares) or saline (combined SA/C groups, triangles) $2 \mathrm{~h}$ before this training session.

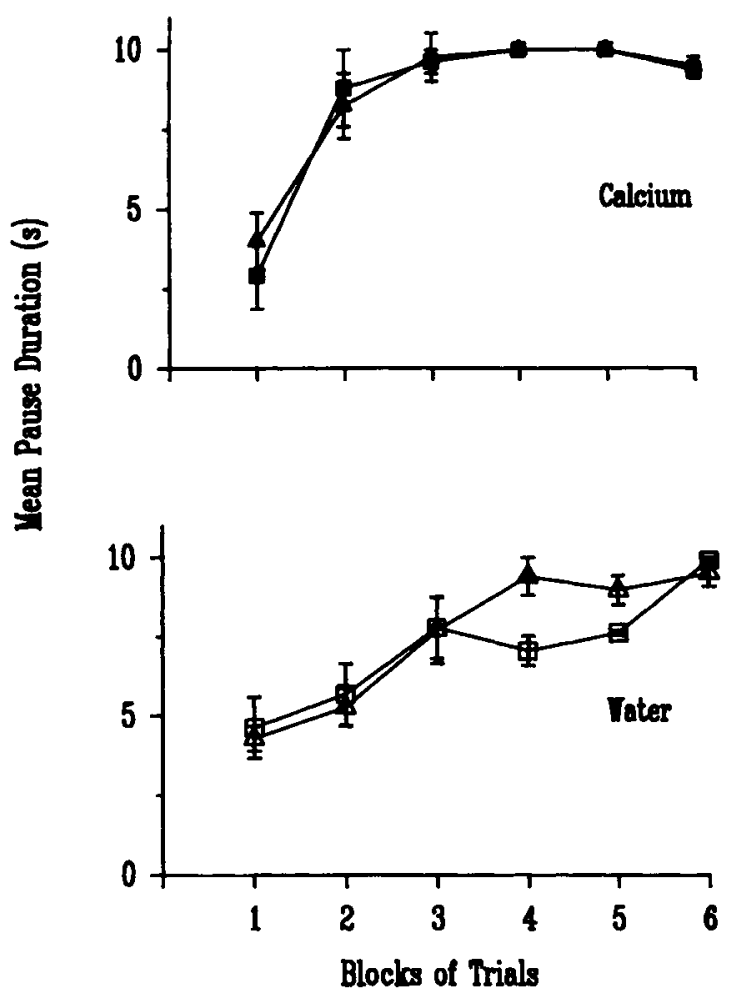

Figure 5. Mean durations of pause responses (CR) before the electrified grid anea over sixths of suppression training trials. Solid symbols represent subjects reared and reinforced with calcium-supplemented water; open symbols represent those reared and reinforced with plain water. Within each fluid treatment, subjects received either injections of $300 \mathrm{mg} / \mathrm{kg}$ salicylate (SB groups, squares) or saline (combined SA/C groups, triangles) $2 \mathrm{~h}$ before this training session.

first block of trials only. Thus, overall trial latencies, exclusive of the CR durations, were rather insensitive to fluid type or salicylate treatment, with the single exception of a weak effect of fluid type at the very beginning of training.

Figure 5 shows the strength of the pause responses (CR) before the shock grid area during sixths of acquisition trials for the treatment groups reared and reinforced with either water or the calcium solution. A salicylate effect may be seen in the lower panel by comparing the pause latencies in Trial Blocks 4 and 5 for those salicylateinjected subjects reared and trained with tap water (open squares) with similarly reared subjects injected with saline (combined SA/C groups, open triangles). In contrast, both calcium groups (upper panel) were virtually identical and had a pattern of overall stronger CRs than did both water-reared groups from Trial Blocks 2-5. It should be noted that the last block of trials contained the criterion latencies of five consecutive trials with pause durations of at least $5 \mathrm{sec}$. Analysis of mean pause responses per six blocks of training revealed a main effect of fluid type $[F(1,32)=10.97, p<.003]$, but there was no main effect of salicylate treatment, nor was there an interaction. The progressive trial-blocks effect $[F(5,160)=$ 
$39.43, p<.0001$ ], generally reflected in Figure 5, indicated the gradual acquisition of the pause response with increased training trials. A block $\times$ fluid-type interaction $[F(5,160)=4.30, p<.002]$ emerged due to consistently longer pause durations in the calcium groups than in the water-reared-and-reinforced groups on all trial blocks (all $p s<.05$ ) except for the last. The block $\times$ salicylatetreatment interaction was marginally significant $[F(5,160)$ $=2.04, p=.08]$, as was the three-way interaction actually depicted in Figure $5[F(10,150)=1.82, p=.08]$. Separate analyses of each block showed significant effects of type of fluid on Trial Blocks 2-5 (all ps $<.01$, at least), but only on Trial Blocks 4 and 5 did clearly significant fluid $X$ salicylate-treatment interactions emerge $[F(1,32)=6.46, p<.02$ and $F(1,32)=4.98, p<.03$, respectively].

Extinction. Two hours prior to the extinction session on the day following training, both the SA and SB groups received $300 \mathrm{mg} / \mathrm{kg}$ sodium salicylate injected s.c. The control groups received second injections of the saline vehicle. Figure 6 shows the mean number of extinction trials for each group. The trials to the extinction criterion of five consecutive nonpause responses (i.e., latencies observed before the previously electrified area that did not exceed $1 \mathrm{sec}$ ) in this session showed only a main effect of salicylate treatment $[F(2,32)=4.86, p<.0149]$. No significant interactions were found. From Figure 6, it appears that, with the single exception of the plain-water control group, trials in extinction were fairly comparable, and the statistical salicylate effect was largely accounted for by the control groups' difference (Duncan test, $p<.05$ ), collapsed across fluid rearing condition, com-

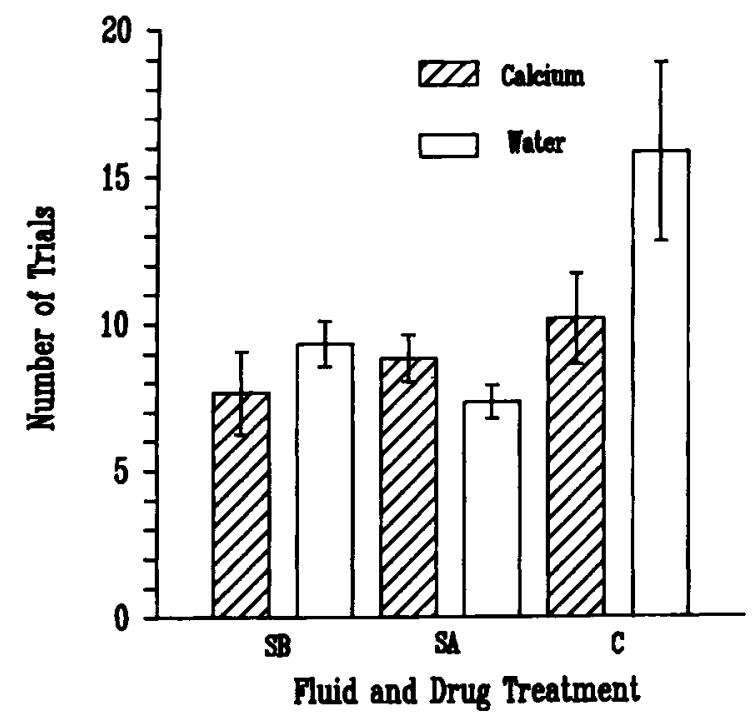

Figure 6. Mean number of trials to extinguish the suppression behavior of pause responses in the groups reared and reinforced with exogenous calcium (slashed blocks) or plain water (open blocks). Four groups received s.c. salicylate injections prior to the extinction session (SB and SA), while the remaining groups received saline injection (C). pared with the two salicylate-injected groups, which were statistically equivalent.

As with acquisition training, the total numbers of extinction trials were divided into blocks, each representing one sixth of the total, and the mean latency per block for each subject was calculated and treated as a data point for the analysis. The alley latency measure proved significant only for trial blocks $[F(5,160)=16.82$, $p<.0001]$, which confirmed the expectation of faster running in all subjects with progressive extinction trials.

Mean durations of the pause responses over blocks of trials in extinction are depicted in Figure 7, indicating fairly comparable and progressive shortening of latencies across sixths of extinction trials in all groups, a trend supported by a significant effect for trial blocks $[F(5,150)=40.57, p<.0001]$. While the main effects of fluid type and salicylate treatment were not significant, their interaction was $[F(2,30)=5.08, p<.02]$. None of the remaining interactions attained acceptable significance. The SB groups under both fluid rearing conditions extinguished with different patterns relative to their respective $\mathrm{SA}$ and $\mathrm{C}$ groups, and the direction of the $\mathrm{SB}$ difference, in turn, was opposite for each fluid treatment. This observation was confirmed by the Duncan tests for comparisons on Trial Block 1, indicating that the SB groups were different from the SA and C groups, which did not differ from each other. Likewise, on Trial Block 5 , the difference between the SB groups and the other groups of the water-reared condition contributed to an overall difference between SB and other groups (all $p$ s<.05).

\section{Discussion}

The similarity of acquisition of the suppression behavior in all groups indicated that the subjects were able to discriminate the CS (i.e., the offset from the background noise), and this detection occurred regardless of the fluid solution available during rearing and reinforcement. The salicylate effect during the suppression training stage (Figure 5) was evident in the measure of CR strength, just after the middle of training, in the comparison between the water-reared-and-reinforced group injected $2 \mathrm{~h}$ prior to training (SB, water) and their combined $\mathrm{SA} / \mathrm{C}$ counterpart group. This finding is intriguing because differences in acquisition of suppression between salicylate and saline groups have not been observed in adults, but this contrast is most readily attributed to differences in the dependent measures employedlick rate with the adults versus response latency in the pups. That a salicylate effect in acquisition was seen affirms the sensitivity of the response-latency measure and suggests that further investigation of adult suppression is needed as well.

The effects of calcium treatment on CR durations during acquisition suggest two possibilities. First, Calcium, in general, may facilitate performance through some kind of energizing capacity; and second, it may offer some protection from salicylate effects. The first possibility is reflected in the overall better CR performance in cal- 


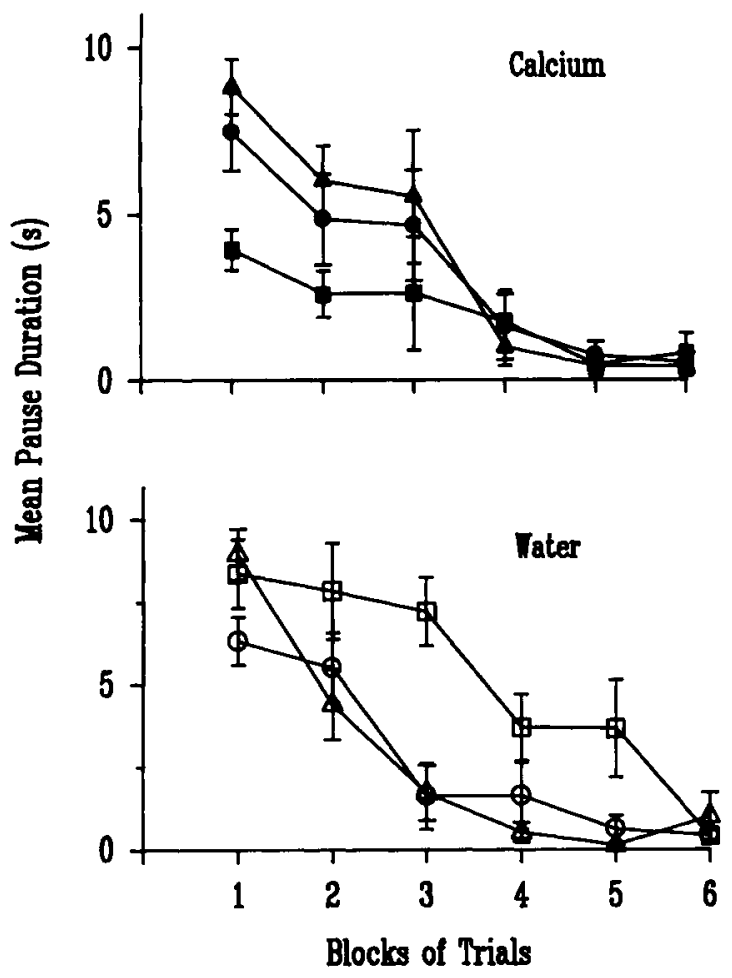

Figure 7. Mean durations during extinction of the pause response before the grid area that had been electrified during acquisition training. Latencies for sixths of extinction trials are plotted for the three groups that received the calcium fluid (solid symbols) or plain tap water (open symbols). The subjects were injected before both acquisition and extinction sessions with the same solution - either salicylate (SB, squares) or saline (C, circles) - or with the saline vehicle before training and salicylate prior to the extinction session (SA, triangles).

cium groups regardless of salicylate treatment. Studies of the role of calcium in the physiopathology of stress suggest that calcium and stress levels covary. For example, De Marino, Pisanti, and Capone (1991) reported that the calcium channel blocker nimodipine attenuated weight loss and eventual mortality in aged (24-month-old) female rats exposed to avoidance training and to inescapable footshocks. Others (e.g., Scrogin, Hatton, \& McCarron, 1991) have found that calcium supplements seem to interact with other metabolic factors in mediating stress responses in hypertensive rats. Thus, it may be that the calcium supplement counteracted stress levels, perhaps by energizing performance through increased attention and sensitivity to conditioning stimuli. The second interpretation suggests specific calcium effects on salicylateinduced phantom auditory perception. Concerning this possibility, if the auditory effect induced by salicylate is the result of a disruption in calcium homeostasis, it is possible that supplementing calcium levels by exogenous ingestion partially blocks or masks this phantom perception. Results from recording of perilymphatic free calcium support this possibility, since while calcium supplementation did not affect the base level of calcium, it did attenuate salicylate-induced decrease of free calcium (Jastreboff et al., 1992).

Another means of influencing subjects' behavior by calcium supplementation is through decreasing the stress level. We have found that $0.05 \mathrm{M}$ exogenous calcium supplement reduced weight loss and instances of mortality at high salicylate doses $(300$ and $350 \mathrm{mg} / \mathrm{kg}$ ) when adult subjects were exposed to auditory stress of $98 \mathrm{~dB}$ (SPL) noise, but not when the source of stress was physical confinement (Jastreboff \& Brennan, 1994a). One explanation of this result suggests that confinement may be more stressful than intense noise, so that the alleviation of salicylate-induced toxicity by calcium supplementation is not enough to prevent mortality. While the mechanisms translating calcium effects on general metabolism, stress levels, and sensory acuity are unclear, it does appear that supplemental calcium in the present context did eliminate salicylate effects in acquisition of the pause CR.

Within the water-reared-and-reinforced groups, the pattern of CR extinction generally conforms to expectations from studies of adult rats (Jastreboff, Brennan, et al., 1988; Jastreboff \& Brennan, 1992) - namely, a perseveration of responding when salicylate administration remained constant from acquisition training to extinction testing. Thus, the perseveration of CR strength in the SB group reared with water conforms to the prediction that the presence of salicylate-induced phantom sound during acquisition training contributes to the resistance to extinction if present during that phase as well. For the SA treatments, the expectation of least resistance to extinction when the injection sequence changed from saline to salicylate, from acquisition to extinction, was only suggested in the comparison with the control group on Trial Block 4. Indeed, the pause-duration data suggest quite comparable responding between SA and $\mathrm{C}$ groups, which may perhaps be due to a floor effect of short latency responses during the last half of extinction testing. One additional note in the control group concerns the relatively short pause duration despite the rather uniquely large number of extinction trials, as seen in Figure 6.

During the first half of extinction trials for the calciumreared-and-reinforced groups (upper panel of Figure 7), the order of mean latencies is the opposite from expectations based on adults. Whereas the SA and $\mathrm{C}$ groups with supplemental calcium are fairly similar, the SB group is consistently faster during the first three blocks of extinction trials. In fact, this difference between the SB and other calcium groups, which was significant on the first two blocks of extinction trials, represents the only apparent salicylate effect in calcium-reared subjects during both acquisition and extinction. The emergence of a salicylate effect in the SB group is intriguing also because if this effect is auditory, it appears to be acting as a novel cue for the SB subjects, in a way that is similar to predictions from adult results that point to the novel value of additional auditory information when salicylate is injected after training, prior to extinction.

The general similarity of pause durations in the SA and $\mathrm{C}$ groups for both rearing fluids during extinction is 
rather striking. As a consequence, it is the SB treatment that mostly distinguishes the pause-duration data among groups during extinction in comparisons between waterand calcium-reared subjects. The results of the waterreared SB group, consistent with those of adult subjects, are readily explained as being due to the presence of salicylate-induced phantom sound perception during acquisition taking on aversive properties, which contributes to resistance to extinction. Given the lack of a salicylate effect in the acquisition measures of the calciumsupplemented subjects, and the general similarity between SA and C calcium groups, it seems unlikely that a specific salicylate effect, such as sound perception, occurred uniquely in the SB group. Similarly, given our extensive use of multiple injections of various doses of salicylate in adult rats (e.g., Jastreboff \& Brennan, 1992) and use of the same dose levels in young rats (e.g., Brennan et al., 1993), the possibility of some vestibular or physiological side effects constituting a cue for the calcium SB group seems remote. However, the only difference between the SB and SA calcium treatments was in the sequence and number of salicylate injections, so this possibility cannot be entirely dismissed.

The purpose of Experiment $1 \mathrm{~B}$ was to test whether calcium enrichment of diet would modify the auditory effects of salicylate in postweanling rats. In both acquisition and extinction, salicylate effects were inferred from performance differences arising from the introduction of salicylate relative to training conditioned suppression. As was the case with adult rats, the time of introduction of the salicylate in the water-reared pups of the present experiment provided a predictor of performance from acquisition to extinction. During acquisition training, the calcium supplement eliminated salicylateinduced performance differences in the CR-latency results of the SB and SA/C groups, which was supported by the comparisons between the SB groups of each fluid type. During the second experimental session, extinction of the pause response showed that calcium effects were evident, although quite differentially, and in a way that was dependent upon when salicylate was introduced. In contrast to the expected trend of salicylate treatment, supplemental calcium eliminated group differences in the direction predicted for salicylate and resulted in some evidence of short latencies in the SB group early on, during the first half of training.

\section{REFERENCES}

BÉKÉsY, G. voN (1960). Experiments in hearing. New York: McGrawHill.

Blumberg, M. S., Efimova, I. V., \& Alberts, J. R. (1992). Ultrasonic vocalizations by rat pups: The primary importance of ambient temperature and the thermal significance of contact comfort. Developmental Psychobiology, 25, 229-250.

Brennan, J. F., Byrnes, J. J., \& Jastreboff, P. J. (1993). Salicylateinduced phantom auditory effects on reinforced behavior in pre- and postweanling rats. Psychobiology, 21, 60-68.

BRENNAN, J. F., \& JASTREBOFF, P. J. (1989). Interaction of salicylate and noise results in mortality in rats. Experientia, 45, 731-734.

Brennan, J. F., \& Jastreboff, P. J. (1991). Generalization of condi- tioned suppression in rats following salicylate induced auditory sensation. Acta Neurobiologiae Experimentalis, 51, 15-27.

Brownell, W. E., Shehata, W. E., \& IMredy, J. P. (1990). Slow electrically and chemically evoked volume changes in guinea pig outer hair cells. In N. Akkas (Ed.), Biomechanics of active movement and deformation of cells (pp. 493-498). Berlin: Springer-Verlag.

BRUGGE, J. (1986). Development of the auditory nerve. In R. N. Aslin (Ed.), Advances in neural and behavioral development (Vol. 2, pp. 73-94). Norwood, NJ: Ablex.

Chen, G. D., \& JASTReboff, P. J. (1995). Salicylate-induced abnormal activity in the inferior colliculus of rats. Hearing Research, 82, 158178.

De Marino, V., Pisanti, N., \& Capone, D. (1991). Effects of nimodipine on psychological-stress situation in aged rats. Acta Neurologica, 13, 410-417.

DurRant, J. D., \& Lovrinic, J. H. (1984). Measurements of sound. In J. P. Butler (Ed.), Bases of hearing science (pp. 52-84). Baltimore: Williams \& Wilkins.

FloCK, A., FlOCK, B., \& UlFENDAhl, M. (1986). Mechanisms of movement in outer hair cells and a possible structural basis. Archives of Otorhinolaryngology, 243, 83-90.

FrIAUf, E. (1992). Tonotopic order in the adult and developing auditory system of the rat as shown by c-fos immunocytochemistry. $E u$ ropean Journal of Neuroscience, 4, 798-812.

Goodwin, G. A., Molina, V. A., \& Spear, L. P. (1994). Repeated exposure of rat pups to isolation attenuates ultrasonic vocalization rates: Reversal with naltrexone. Developmental Psychobiology, 27, 53-64.

Hofer, M. A., Brunelli, S. A., \& Shatr, H. N. (1993). Ultrasonic vocalization responses of rat pups to acute separation and contact comfort do not depend on maternal thermal cues. Developmental Psychobiology, 26, 81-95.

HudSPETH, A. J. (1986). The ionic channels of a vertebrate hair cell. Hearing Research, 22, $21-27$.

HYsON, R. L., \& RuDY, J. W. (1984). Ontogenesis of learning: II. Variation in the rat's reflexive and learned responses to acoustic stimulation. Developmental Psychobiology, 17, 263-283.

JASTREBOfF, P. J. (1990). Phantom auditory perception (tinnitus) mechanisms of generation and perception. Neuroscience Research, 8 , 221-254.

JASTREBOFF, P. J., \& BRENNAN, J. F. (1992). Animal model of tinnitus: Recent developments. In J.-M. Aran \& R. Dauman (Eds.), Proceedings of the Fourth International Tinnitus Seminar, Bordeaux, 1991 (pp. 283-292). Amsterdam: Kugler.

JASTREBOFF, P. J., \& BRENNAN, J. F. (1994a). Alleviation of mortality induced by salicylate and stress. Experientia, 50, 469-474.

JASTREBOFF, P. J., \& BRENNAN, J. F. (1994b). Evaluating the loudness of phantom auditory perception (tinnitus) in rats. Audiology, 33, 202-217.

Jastreboff, P. J., Brennan, J. F., Coleman, J. K., \& Sasaki, C. T. (1988). Phantom auditory sensation in rats: An animal model of tinnitus. Behavioral Neuroscience, 102, 811-822.

JastrebofF, P. J., Brennan, J. F., \& SASAKI, C. T. (1987). Behavioral and electrophysiological animal model of tinnitus. In H. Feldmann (Ed.), Proceedings of the Third International Tinnitus Seminar, Muenster, 1987 (pp. 95-99). Karlsruhe, Germany: Harsch Verlag.

JASTREBOFF, P. J., BRENNAN, J. F., \& SASAKI, C. T. (1991). Quinine induced tinnitus in rats. Archives of Otolaryngology-Head \& Neck Surgery, 117, 1162-1166.

Jastreboff, P. J., Issing, W., Brennan, J. F., \& Sasaki, C. T. (1988). Pigmentation, anesthesia, behavioral factors, and salicylate uptake. Archives of Otolaryngology-Head \& Neck Surgery, 114, 186-191.

JastrebofF, P. J., NguYen, Q., Brennan, J. F., \& Sasakı, C. T. (1992). Calcium and calcium channel involvement in tinnitus. In J.-M. Aran \& R. Dauman (Eds.), Proceedings of the Fourth International Tinnitus Seminar, Bordeaux, 1991 (pp. 109-114). Amsterdam: Kugler.

KeLLY, J. B. (1990). Rat auditory cortex. In B. Kolb \& R. C.Tees (Eds.), The cerebral cortex of the rat (pp. 381-405). Boston: MIT Press. KRONESTER-FrEI, A. (1979). The effect of changes in endolymphatic ion concentrations on the tectorial membrane. Hearing Research, 1 , $81-94$.

RUBEL, E. W. (1978). Ontogeny of structure and function in the verte- 
brate auditory system. In M. Jacobson (Ed.), Handbook of sensory physiology (pp. 135-237). Berlin: Springer-Verlag.

RUBEL, E. W. (1984). Ontogeny of auditory system development. Annual Review of Physiology, 46, 213-229.

Rubel, E. W., Hyson, R. L., \& Durham, D. (1990). Afferent regulating of neurons in the brain stem auditory system. Journal of Neurobiology, 21, 169-196.

RUDY, J. W., \& HYSON, R. L. (1982). Consummatory response conditioning as an auditory stimulus in neonatal rats. Behavioral Neural Biology, 34, 209-214.

RuDY, J. W., \& HySON, R. L. (1984). Ontogenesis of learning: III. Variation in the rats' differential reflexes and learned responses to sound frequencies. Developmental Psychobiology, 17, 285-300.
Sales, G. D., \& Smith, J. C. (1978). Comparative studies of the ultrasonic calls of infant murid rodents. Developmental Psychobiology, 11, 595-610.

Scrogin, K. E., Hatton, D. C., \& McCarron, D. A. (1991). Effects of dietary sodium and calcium on blood pressure reactivity in the SHR and WKY. Clinical Experimental Hypertension, 13, 699-707.

WINER, B. J. (1971). Statistical principles in experimental design (2nd ed.). New York: McGraw-Hill.

(Manuscript received January 31, 1995; revision accepted for publication May 11, 1995.) 